\title{
Dense high-energy -rays emission by ultra-intense laser interacting with a concave target
}

DOI:

10.1063/5.0010761

\section{Document Version}

Accepted author manuscript

Link to publication record in Manchester Research Explorer

\section{Citation for published version (APA):}

Zhao, Y., Liu, J., Xia, G., \& Bonatto, A. (2020). Dense high-energy -rays emission by ultra-intense laser interacting with a concave target. Physics of Plasmas, 27(7), 073106. https://doi.org/10.1063/5.0010761

\section{Published in:}

Physics of Plasmas

\section{Citing this paper}

Please note that where the full-text provided on Manchester Research Explorer is the Author Accepted Manuscript or Proof version this may differ from the final Published version. If citing, it is advised that you check and use the publisher's definitive version.

\section{General rights}

Copyright and moral rights for the publications made accessible in the Research Explorer are retained by the authors and/or other copyright owners and it is a condition of accessing publications that users recognise and abide by the legal requirements associated with these rights.

\section{Takedown policy}

If you believe that this document breaches copyright please refer to the University of Manchester's Takedown Procedures [http://man.ac.uk/04Y6Bo] or contact uml.scholarlycommunications@manchester.ac.uk providing relevant details, so we can investigate your claim.

\section{OPEN ACCESS}


Dense high-energy $\gamma$-rays emission by ultra-intense laser interacting with a concave target

\author{
Dense high-energy $\gamma$-rays emission by ultra-intense laser interacting with a \\ concave target \\ Yuan Zhao, ${ }^{1, \text { a) }}$ Jianxun Liu, ${ }^{2}$ Guoxing Xia, ${ }^{1,3, b)}$ and Alexandre Bonatto ${ }^{1,3}$ \\ 1) Department of Physics and Astronomy, University of Manchester, Manchester M13 9PL, \\ $U K$ \\ ${ }^{2)}$ Wuhan Electronic Information Institute, Wuhan 430019, China \\ ${ }^{3)}$ Cockcroft Institute, Daresbury WA4 4AD, UK \\ (Dated: 27 June 2020)
}

We propose to use a 10 petawatts (PW) laser irradiating onto a target with a concave surface, which can focus the laser beam and attain a more intense laser field, so as to increase both the yield and mean energy of emitted $\gamma$-rays. 2D PIC simulation results show that the peak electric field after the reflection of laser from the target in this new scheme can reach $\sim 1.8$ times as high as that in the plane target case. Such an enhanced laser field enables an increase of the number of hot electrons in front of the incident plane and raises the probability rate of generating high-energy $\gamma$-rays significantly by the non-linear Compton scattering. As a result, $1.5 \times 10^{13} \gamma$ photons with a mean energy of $9.2 \mathrm{MeV}$ are obtained. These photons mainly point toward two directions and distribute in pulses with the duration of each pulse of $\sim 400$ as. A brightness as high as $3.6 \times 10^{25}$ photons $/\left(\mathrm{mm}^{2} \mathrm{mrad}^{2} \mathrm{~s} 0.1 \% \mathrm{BW}\right)$ is obtained in one pulse. Compared to no positron observed for a plane target, a positron yield of $3.0 \times 10^{8}$ can be achieved in this new scheme.

\section{INTRODUCTION}

$\gamma$-ray sources via laser-plasma interaction have attracted lots of attention for their high energy, short duration and high brightness ${ }^{1-4}$. In this aspect, the cutting-edge PW laser facilities have played a key role in advancing this technology. Current physics models for $\gamma$-rays generation by ultrahigh laser interacting with plasma include skin-depth emission ${ }^{5}$, reinjected electron synchrotron emission (RESE) ${ }^{6,7}$, non-linear Compton scattering and synchrotron emission from electron oscillation in different electric and magnetic fields ${ }^{8-11}$, such as electromagnetic modes in microplasma waveguide ${ }^{12,13}$, selfgenerated magntic field ${ }^{2}$ and laser field ${ }^{14}$. The skin-depth emission is mainly from laser-solid interaction, which involves effects like relativistic hole boring, skin effect and relativistic transparency. While the RESE regime is more dominant in underdense plasma. Synchrotron radiation is competitive in special field, while it is negligible when concerning high energy photons, especially in counter-propagating laser fields, where the probability rate of non-linear Compton scattering is significantly enhanced ${ }^{15}$. The required counterpropagating laser beams can be realized by overlapping one laser pulse with its reflected pulse by striking a solid surface or using two colliding beams ${ }^{16-18}$. The second way is particularly efficient in laser-gas interaction ${ }^{19}$. Simulations from Ridgers et al. ${ }^{5}$ have indicated that laser-solid interaction can get more high-energy photons and positrons compared to laser-gas interaction when the laser intensity is lower than $8 \times 10^{23} \mathrm{Wcm}^{-2}$, although the generation rate is lower than that from gas target. Thus using laser-solid interaction is the most efficient way to generate dense and high-energy photons and positrons under the laser intensity that can be obtained in labs nowadays ${ }^{20}$. Generally, a foil is struck by an intense

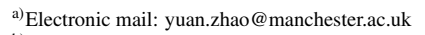

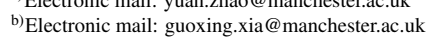

laser to generate dense $\gamma$-rays and positrons $s^{3,21}$. The colliding lasers are utilized to improve the production of photons and positrons ${ }^{18,22}$. In particular, Zhang et al. obtained high yield ${ }_{20}$ of photons by adjusting the delay time between two lasers to make the electrons are accelerated by light pressure ${ }^{22}$. However, the control of delay time between two lasers requires further advancement with the laser technique. Moreover, the energy conversion efficiencies from laser to photons or positrons ${ }_{25}$ are relatively low ${ }^{23,24}$. Therefore, it is essential to optimize the shape of the solid target to enhance the conversion process.

In QED regime, for electrons with relativistic factor of $\gamma_{e}$, the differential probability rate of photon emissions is given by 25,26 :

30

$$
\frac{d W_{\gamma}}{d \chi}=\frac{\sqrt{3} \alpha}{2 \pi \tau_{C}} \frac{\eta}{\gamma_{e}} \frac{F(\eta, \chi)}{\chi}
$$

where $\alpha$ is the fine structure constant, $\tau_{C}=\hbar / m_{e} c^{2}=1.288 \times$ ${ }_{35} 10^{-6} \mathrm{fs}$ is the Compton time, $\hbar$ is reduced Planck constant, $m_{e}$ is electron rest mass, $c$ is the speed of light in vacuum, $F(\eta, \chi)=\frac{4 \chi}{3 \eta^{2}}\left[\left(1-\frac{2 \chi}{\eta}+\frac{1}{1-2 \chi / \eta}\right) K_{2 / 3}(\delta)-\int_{\delta}^{\infty} K_{1 / 3}(t) d t\right]$ is the quantum synchrotron function ${ }^{27}$, where $\delta=\frac{4 \chi}{3 \eta^{2}}(1-$ $\left.{ }_{40} \frac{2 \chi}{\eta}\right)^{-1}, K_{1 / 3}$ and $K_{2 / 3}$ are modified Bessel functions. The most important parameters in Equation (1) are $\eta=\gamma_{e} \mid \vec{E}_{\perp}+$ $\vec{v} \times \vec{B} \mid / E_{\text {sch }}$ and $\chi=\hbar\left|\omega \vec{E}_{\perp}+c^{2} \vec{k} \times \vec{B}\right| / 2 m_{e} c^{2} E_{s c h}{ }^{28,29}$. They are Lorentz invariants, used to describe the transition rates from electrons to photons and photons to positrons respec${ }^{4}$ tively, where $\overrightarrow{E_{\perp}}$ is the electric field that is perpendicular to the velocity of electrons $\vec{v}$ or wavevector of photons $\vec{k}$, $E_{s c h}=1.3 \times 10^{18} \mathrm{~V} / \mathrm{m}$ is the Schwinger field, $\vec{B}$ is the magnetic field strength and $\omega$ is the frequency of photons. In order to demonstrate the effect of the electric field on the generation of photons, the velocity of electrons $\vec{v}$ is assumed to be perpendicular to the electric field. Integrating Equation (1) over $\chi_{0} \leq \chi \leq \eta / 2$, where $\chi_{0}$ is dominant by the lower limit of the energy of photons that needs to be counted, gives the rates of production for photons with energy $E_{\gamma}>1 \mathrm{MeV}$ and 


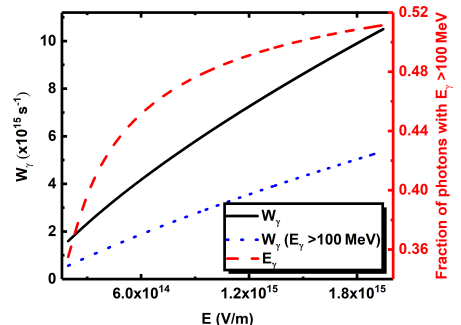

FIG. 1. Probability rate of generation for $\gamma$-rays over all photons energies (black solid line) and photons energy greater than $100 \mathrm{MeV}$ (blue dot line) emissions versus intensity of electric field. The right axis shows the fraction of $\gamma$-rays with energy higher than $100 \mathrm{MeV}$.

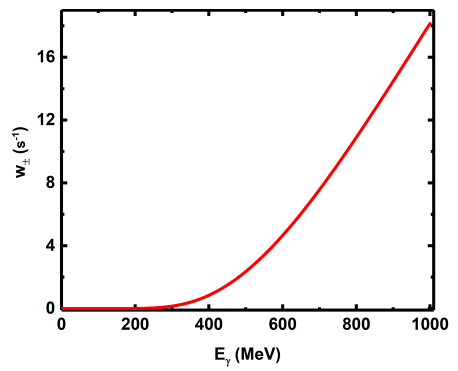

FIG. 2. Probability rate of positron emission versus the energy of photons that emitting positrons.

$E_{\gamma}>100 \mathrm{MeV}$ respectively. The rates for both of them are plotted in Figure 1, which indicates that the yield of high-

85 energy photons increases with the laser field.

The rate of positron emission is described as ${ }^{25}$ :

$$
W_{ \pm}=\frac{\alpha}{\tau_{C}} \frac{m_{e} c^{2}}{\hbar \omega} \chi T(\chi)
$$

where $\hbar \omega$ is the energy of photons that emitting positrons and 40 $T(\chi)$ is a function of $\chi^{30}$ :

$$
T(\chi)= \begin{cases}\frac{3 \sqrt{3}}{8 \sqrt{2}} \exp \left(-\frac{4}{3 \chi}\right), & \chi \ll 1 \\ 0.6 \chi^{-1 / 3}, & \chi \gg 1\end{cases}
$$

9o Figure 2 reveals that the probability rate of positron-electron pair production increases exponentially with photon energies Therefore, intenser laser field can boost the high-energy photon and positron emissions consequently.

95 In this paper, we propose to utilize a concave structure intso the front surface of the foil to enhance the emissions of highenergy photons and positrons. Due to the focus function of the concave surface, both the electric and magnetic fields are enhanced enormously, which leads to more hot electrons in front ${ }_{55}$

100 of the target. These electrons colliding with laser photons can generate more high-energy photons by non-linear Compton scattering ${ }^{31}$ and create more positrons via Breit-Wheeler process $^{32}$. We analyse the enhancement of laser fields theoretically and simulate the interaction of laser with proposed

105 concave target by Particle-in-Cell (PIC) code. $\gamma$-rays as dense as $\sim 1.0 \times 10^{30} \mathrm{~m}^{-3}$ are emitted with the mean energy of 9.2 MeV. Consequently, the yield of $\gamma$-rays is $1.6 \times 10^{13}$, which is several orders of magnitude high than that of the plane target. This paper is arranged as follows. In Sec. II,

110 we describe the details of simulation and analyse the reasons of enhanced $\gamma$-ray emissions. Then, we discuss result dependence on geometric parameters in Sec. III. Sec. IV gives the conclusions.

\section{SIMULATION}

${ }_{115}$ We perform a 2D simulation with widely used QED$\mathrm{PIC}$ code $\mathrm{EPOCH}^{33-35}$. Figure 3 displays the schematic of the proposed structure. The laser pulse is circularly polarized with dimensionless amplitude following profile $a=a_{0} \exp \left(-y^{2} / \sigma^{2}\right) \exp \left(-t^{2} / \tau^{2}\right)$, where $a_{0}=191$ is the

120 peak amplitude, corresponding to the peak intensity of $1 \times$ $10^{23} \mathrm{Wcm}^{-2}, \sigma=4.0 \mu \mathrm{m}$ is the waist radius of this Gaussian beam and $\tau=10 T_{0}$ is the width of the pulse with the period $T_{0}=3.33 \mathrm{fs}$. The wavelength of the laser is $\lambda_{0}=1 \mu \mathrm{m}$, which corresponds to the critical density of $n_{c}=4 \pi^{2} c^{2} \varepsilon_{0} m_{e} / \lambda_{0}^{2} e^{2}=$

$1251.1 \times 10^{27} \mathrm{~m}^{-3}$. The constants in this expression are vacuum permittivity $\varepsilon_{0}$ and the elementary charge $e$, respectively. The target is solid aluminium with electron density of $n_{e}=500 n_{c}$ and it is located in the region $7 \mu \mathrm{m} \leq x \leq 9.7 \mu \mathrm{m}$. Two key structural parameters for this scheme are the curvature of the 3o concavity $R=1.5 \mu \mathrm{m}$ and the distance from the center of the concave surface to the left boundary of the target $d_{0}=0$. Besides, the thickness of the target behind the concave surface is $d_{1}=1.2 \mu \mathrm{m}$, which has no effect on the interaction provided that it is thick enough that the laser cannot pass 5 through it. Moreover, the simulation box is $20 \mu \mathrm{m} \times 20 \mu \mathrm{m}$, divided into $1800 \times 1800$ grids, namely the size of each cell is $0.011 \mu \mathrm{m}$. Generally, the size of grid should be shorter than both the Debye length $\lambda_{D}=\sqrt{\varepsilon_{0} k_{B} T_{e} / n_{e} e^{2}}$ and the skin depth $\delta=c / \omega_{p}$, where $\omega_{p}=\sqrt{n_{e} e^{2} / \varepsilon_{0} m_{e}}$ is the electron plasma frequency, $T_{e}$ is the temperature of electrons, $k_{B}$ is the Boltzmann constant ${ }^{36}$. Here, each grid involves 42 pseudopaticles. These particles distribute with a triangle shape function ${ }^{33}$. Since the simulation focuses on $\gamma$-ray and positron emissions, only the photons with energy $E_{\gamma}>1 \mathrm{MeV}$ are counted. The

45 radiation reaction effect is also taken into account because of the intense laser intensity ${ }^{37}$. In order to show the significant enhancements of high-energy photons and positrons, the plane foil case, which is normally used to generate positrons is simulated as a comparison group. The laser and target parameters for these two cases are the same. For the thickness of the foil, it is set to $2 \mu \mathrm{m}$ aiming to eliminate its effect on final result.

Figure 4 shows the spatial distributions of both transverse electric and magnetic fields at $10 T_{0}$, corresponding to the time that the peak electric field occurs. The units of them are the amplitudes of electric and magnetic fields for the simulated laser, namely $E_{0}=6.2 \times 10^{14} \mathrm{~V} / \mathrm{m}$ and $B_{0}=2.0 \times 10^{6} \mathrm{~T}$, respectively. When the laser interacts with a plane target, sim- 


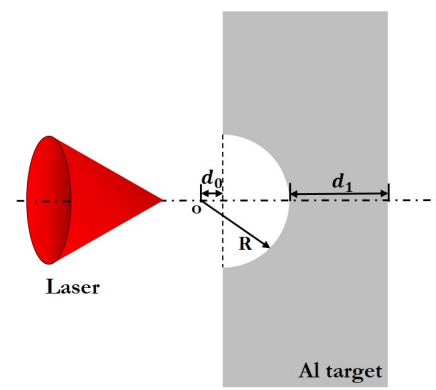

FIG. 3. Schametic of the concave target shining by a PW laser beam. The grey part is the aluminium target with electron density of $500 n_{c}$. The point $o$ is the center of the concavity, $R$ is the curvature of the concave surface, $d_{0}$ is the distance between the center and the left boundary of the target and $d_{1}$ represents the thickness of the target behind the concave. The red cone is the circularly polarized Gaussian laser with intensity of $1 \times 10^{23} \mathrm{Wcm}^{-2}$.

ulations show that the fields increase a little, i. e., $1.6 E_{0}$ and $1.2 B_{0}$ because of the relatively weaker superposition of incident and reflected laser beams. However, in the new scheme, as shown in Figure 4, the maxima of electric and magnetic fields can reach up to $\sim 2.8 E_{0}$ and $\sim 4.3 B_{0}$, respectively. These enhancements are owing to the improved superposition between the incident and reflected pulses from the concave surface. From the interference theory, the incident and reflected laser rays are coherent when the phase difference between them are $2 n \pi(n=0,1,2, \ldots)$. Geometrical analysis gives the optical path difference between incident and reflected rays as $\Delta x=\Delta y \tan \theta+\lambda_{0} / 2$, where $\theta$ is the central angle of incident light and $\Delta y$ means the transverse distance between the incident and reflected lasers. For the focal size of laser used in these simulations, namely $\sigma=4 \mu \mathrm{m}$ and $R=1.5 \mu \mathrm{m}$, corresponding to $0<\Delta y \leq 2 R$, which can guarantee the happening of enhancement at part of the concave surface. As the angle between the incident and reflected rays is $2 \theta$, the final interfered laser field can be derived from $E_{l}^{2}=E_{i}^{2}+E_{r}^{2}+2 E_{i} E_{r} \cos 2 \theta \cos \Delta \phi$. Assuming that $E_{i}=E_{r}=E_{0}$, then $E_{l \max }=2.8 E_{0}$, which agrees well with the simulation results showed above. These increasing fields benefit both the $\gamma$-ray and positron emissions. In our regime, the $\gamma$-rays are mainly emitted by non-linear Compton scattering process between the hot electrons in front of the concave surface and the enhanced laser field. In fact, in this new structured target, the laser keeps dragging out electrons after it starts interacting with the left boundary. This leads to the more efficient absorption of laser energy compared with general plane foil. As a result, the energy of electrons in front of the concave surface is greatly increased. In addition, both the number of electrons participating this interaction and the scattering zone are improved in the new concavity scheme compared to the plane target. Based on these beneficial factors, a higher $\eta \sim 0.04$, which is only $\sim 1 \times 10^{-3}$ in plane target, can
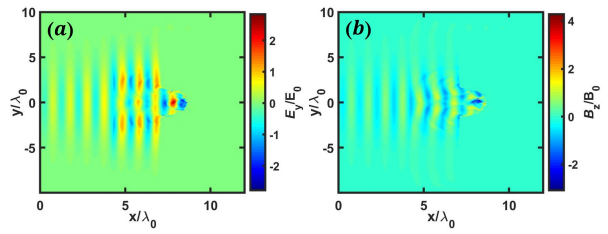

FIG. 4. Distributions of both transverse electric (a) and magnetic (b) fields at $10 T_{0}$. The units $E_{0}$ and $B_{0}$ are the amplitudes of electric and magnetic fields for the laser used in the simulation, which corresponds to $6.2 \times 10^{14} \mathrm{~V} / \mathrm{m}$ and $2.0 \times 10^{6} \mathrm{~T}$ respectively.

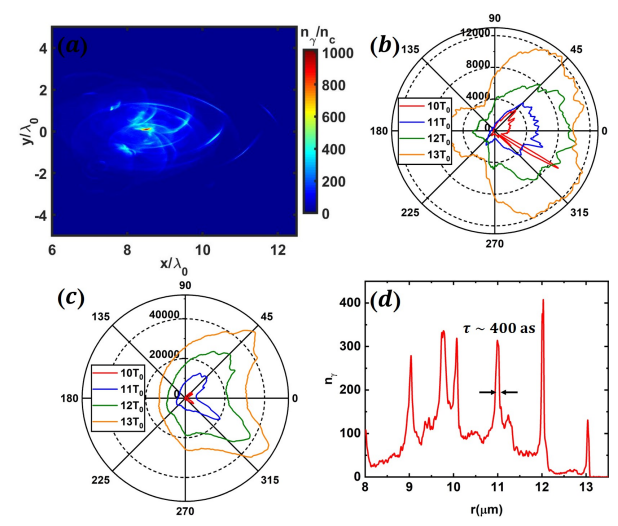

FIG. 5. The density (a) and angular distribution (c) of emitted $\gamma$ photons at $13 T_{0}$ before dense positrons are generated. (b) is the angular distribution of electrons with Lorentz factor $\gamma_{e}>10$. (d) is the $\gamma$ ${ }_{17}$ pulses along the direction of $\theta=-30.5^{\circ}$, which is the angle corresponding to the peak intensity.

be achieved and the probabilities of generating photons and 17galso high-energy photons consequently increase. As shown in

Figure 5 , the density of photons is as high as $\sim 1000 n_{c}$, which is only less than $10 n_{c}$ with thin foil target. The angular distribution of the radiation is given in Figure 5 (c). There are two peaks of radiation intensity at angles $\theta \approx 45^{\circ},-30.5^{\circ}$. These ${ }_{18 a}$ are due to the electron bunches, that are expelled out and accelerated when the laser traverse the left boundary, which are displayed in Figure 5 (b). The gamma pulses at $\theta=-30.5^{\circ}$ is drawn in Figure 5 (d). It is found that the duration of $\gamma$ pulses are $\sim 400$ as.

185 The yields of both photons and positrons, which are displayed in Figure 6, over a time period from $8 T_{0}$ to $20 T_{0}$. Here, $8 T_{0}$ is the time at which the laser beam starts being reflected by the target. Here it is worth pointing out that the 2D simulation sets the $3 \mathrm{rd}$ dimension as $1 \mathrm{~m}$. So the yield in $2 \mathrm{D}$ ${ }_{19}$ should multiply the size of the laser to eliminate this effect. It is obvious that the emissions of photons and positrons grow dramatically after reflection, corresponding to the start of the non-linear Compton scattering. This process keeps occurring

\section{5 \\ 205} 210 215 


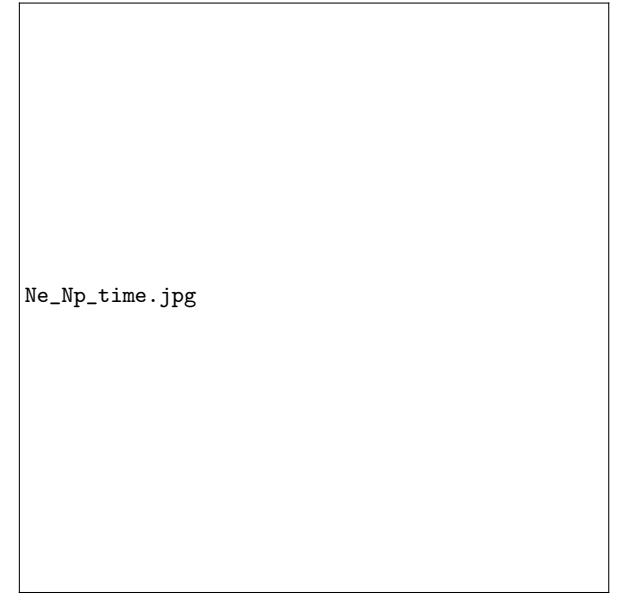

FIG. 6. The evolutions of the numbers of emitted photons $N_{\gamma}$ (a) (black solid line) and positrons $\mathrm{N}_{\mathrm{e}+}$ (b). (a) also displays the number of photons with energy $E_{\gamma}>100 \mathrm{MeV}$ for this new concave target case and $N_{\gamma}$ for the plane target case, labelled as $\mathrm{N}_{\gamma}\left(\mathrm{E}_{\gamma}>100 \mathrm{MeV}\right)^{2}$ (blue dash line) and $N_{\gamma}$ (Plane Target) (yellow dot line). The red short dash line correlates to the right $y$ axis, displaying the average photon energy.

until $t=14 T_{0}$, when the density of surface electrons in front of the target is too low to generate high energy $\gamma$-rays. That is the reason why the yield of $\gamma$-rays with energy $>100 \mathrm{MeV}$ is stable after $14 T_{0}$, as shown in Figure 6 . The evolution of the

220 mean energy of $\gamma$-rays confirms this since it drops when there is almost no non-linear Compton scattering happening. The peak average photon energy is greater than $10 \mathrm{MeV}$, which ${ }_{26}$ indicates that this regime can be a useful way to create highenergy $\gamma$-ray sources. Furthermore, the number of positrons

225 emitted keeps constant when $t>14 T_{0}$ as well.

The spectra of electrons and emitted photons are illustrated in Figure 7. From Figure 7 (a), electrons are heated ${ }_{70}$ to $\sim 600 \mathrm{MeV}$ with the absorption of laser energy. This is

230 different from the plane target case, where the cut-off energy of electrons is limited to tens of $\mathrm{MeV}$. As mentioned before, the main reason is that such a concave shape is favourable to the deposition of the laser energy. After the laser pulses 2 further propagation, the high energy part of the electron spec-

235 trum decreases because of the radiation reaction, while the high energy part of photons increases obviously as shown in Figure 7 (b). That proves the impact of non-linear Compton scattering process. By integrating these spectra, the kizso netic energies of electrons and photons can be calculated, 240 which are $8.8 \times 10^{6} \mathrm{~J}$ and $4.0 \times 10^{6} \mathrm{~J}$ at $16 T_{0}$, respectively. The corresponding conversion efficiencies from laser to these two energies are $8.4 \%$ and $3.8 \%$. Finally, the yields of photons with energy $E_{\gamma}>1 \mathrm{MeV}$ and $E_{\gamma}>100 \mathrm{MeV}$ are $_{28}$ $1.5 \times 10^{13}$ and $1.1 \times 10^{11}$, which are $4.6 \times 10^{8}$ and 0 for the 245 plane target case, respectively. Undoubtedly, the generation

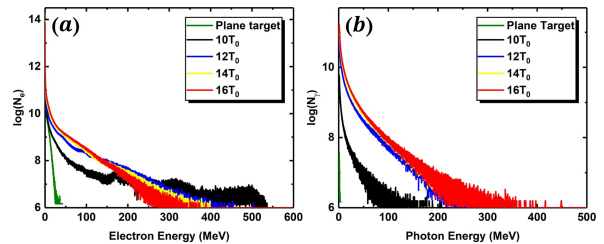

FIG. 7. Spectra of electrons (a) and photons (b) at $10 T_{0}$ (black), $12 T_{0}$ (blue), $14 T_{0}$ (yellow) and $16 T_{0}$ (red), respectively. The spectra for plane target case are also given with dark green line in each pictures.

of high energy band boosts dramatically in this concave target scheme compared with the plane target. Taking that marked pulse in Figure 5 (d) into account, the number of photons is summed as $3.0 \times 10^{11}$ with duration of 433 as, beam size of

$250 \quad 0.13 \mu \mathrm{m} \times 1 \mu \mathrm{m}$ and divergence of $22^{\circ}$. The brightness of this pulse is $3.6 \times 10^{25}$ photons $/\left(\mathrm{mm}^{2} \mathrm{mrad}^{2} \mathrm{~s} 0.1 \% \mathrm{BW}\right)$. Such an improved $\gamma$-ray pulses are beneficial to positron generations. Simulations show that the positrons emitted are $1.3 n_{c}$. Meanwhile, the interaction zone of laser and high-energy photons enlarges significantly due to curved surface. Hence, the yield of positrons reaches $3 \times 10^{8}$, while there is almost no positron generation in laser impinging on a plane target with the laser intensity of $10^{23} \mathrm{~W} / \mathrm{cm}^{2}$. The attained dense high energy density $\gamma$ photons and positrons are useful for investigation of astrophysics phenomena such as black hole, gravitational collapse and gamma-rays burst $^{38,39}$.

\section{DISCUSSION}

When the shape of such a concavity changes, the reflection of laser pulse will be different. This can influence the final electromagnetic field for the generations of $\gamma$-rays and positrons. Thus it is worthwhile to discuss the impact of parameters that may change the geometry of illuminated surface. Figure 8 (a) provides the dependence of positron yield on concave radius when other two parameters are fixed to $d_{0}=0$ and $d_{1}=1.2 \mu \mathrm{m}$. It shows that the yield of positrons peaks at $R=1.5 \mu \mathrm{m}$. The theory described above has already demonstrated that the enhancement of positron emission is mainly caused by the increased high-energy $\gamma$-rays emission, which can be obtained in a more intense laser field. The best concave radius can be derived from the condition that all the light rays meet the coherent condition, which is $\cos 2 \theta \cos \Delta \phi>1 / 2$. That is the main reason why the positrons emission is maximum at $R=1.5 \mu \mathrm{m}$. The number of photons with energy $E_{\gamma}>100 \mathrm{MeV}$, shown in Figure 8 (a), behaves similarly with the curves in Figure 8 (b), which agrees well with the theoretical results.

The distance from the concave centre to the left boundary of the target, i.e. $d_{0}$, can also affect the reflection of the laser field. As $d_{0}$ increases, namely the centre of concave moving towards the target, the transverse length of laser field that interacting with the concave surface is expressed as 


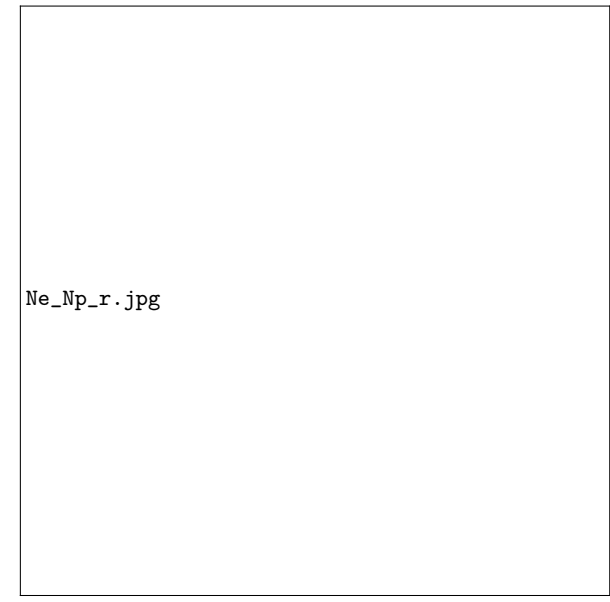

FIG. 8. The influence of concave radius on the sum numbers of emitted photons $N_{\gamma}$ (a) (black solid line) and positrons $N_{e+}$ (b). (a) also displays the yield of photons with energy $E_{\gamma}>100 \mathrm{MeV}$ (blue dash line). The red short dash line connects with the right $y$ axis, providing the mean photon energy versus radius.

$\Delta L=2 \sqrt{R^{2}-d_{0}^{2}}$. As a result, the peak fields drop with $d_{0}$ increasing and then, as shown in Figure 9, the yields of positrons and high-energy photons fall as well.

It is essential to discuss the positron emission under different laser intensities as well. Figure 10 (a) provides the yields of photons and high-energy band photons as a function of laser intensity. The number of emitted photons grows with the laser intensity, resulting in a rising trend of the mean energy for photons. The yield of positrons in Figure 10 (b) behaves similarly to that of high-energy photons. Assuming the energy of photons is constant and $\vec{k}$ is perpendicular to $\vec{B}$, then the rate in Equation (2) is only related to parameter $\chi$. Taking the $\operatorname{logarithm}$ of the probability rate, $\lg W_{ \pm}=A \lg \chi+B$, where $B=\lg \left(\frac{\alpha}{\tau_{C}} \frac{m_{e} c^{2}}{\hbar \omega}\right)$ is a constant while $A$ is the power of $\chi$. From Equation (3), the power of $\chi$ decrease from $\sim 14$ to $2 / 3$ with the increase of $\chi$. Since $\chi \propto E \propto I^{1 / 2}$, it can be derived that $\lg W_{ \pm}=A / 2 \lg I$. Thus the varying rate in Figure 10 decreases as the increase of $\lg I$.

\section{CONCLUSION}

Using a $10 \mathrm{PW}$ laser irradiating onto a target with a concave structure, the laser fields can be enhanced obviously due to the focusing function of the concave on laser field and the enlarg ing zone of laser-electron interaction in front of the target. In consequence, the generation of high-energy $\gamma$-rays is boosted significantly, increasing the yield of positron-electron pairs The results indicate that $\sim 1.5 \times 10^{13} \gamma$ photons are obtained in the concave scheme. They distributes mainly in two direc-

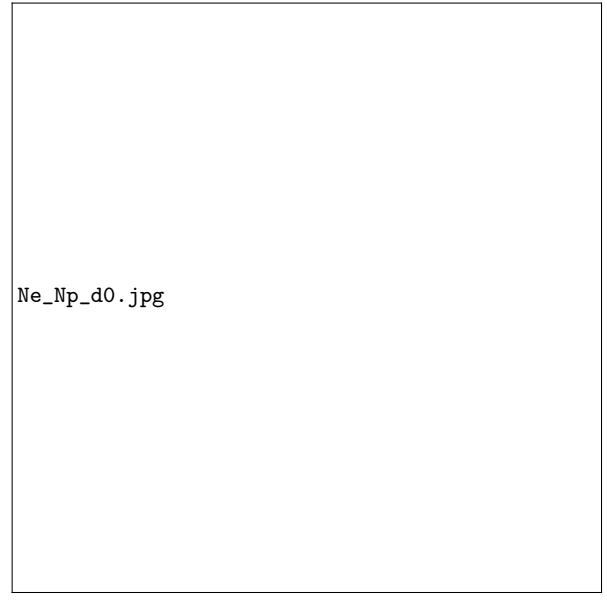

FIG. 9. The effects of $d_{0}$ on yield of photons $N_{\gamma}$ (a) (black solid line) and positrons $\mathrm{N}_{\mathrm{e}+}$ (b). The blue dash line in (a) is the yield of photons with energy $E_{\gamma}>100 \mathrm{MeV}$. The red short dash line associates with the right $y$ axis, corresponding to the average photon energy.

tions with a train of pulses. The brightness of each pulse is as high as $3.6 \times 10^{25}$ photons $/\left(\mathrm{mm}^{2} \mathrm{mrad}^{2} \mathrm{~s} 0.1 \% \mathrm{BW}\right)$. Besides, with these dense high energy photons, this structure attains a yield of $\sim 3 \times 10^{8}$ positrons. This scheme enables the realization of the high flux gamma-ray and position sources using PW laser facilities.

\section{${ }^{295}$ ACKNOWLEDGMENT}

This work was supported by the Presidents Doctoral Scholarship Award of The University of Manchester, the Cockcroft sodnstitute core grant and the STFC. The authors greatly appreciate the computing time from the SCARF cluster of the STFC and the group from University of Warwick that developed the code EPOCH. Contribution from Jianxun Liu was supported by the National Natural Science Foundation (Grant No. 11805278).

\section{DATA AVAILABILITY}

The data that support the findings of this study are available from the corresponding authors upon reasonable request.

310

REFERENCES

${ }^{1}$ Corde S, Phuoc K T, Lambert G, Fitour R, Malka V, Rousse A, Beck A and 315 Lefebvre E 2013 Reviews of Modern Physics 851

${ }^{2}$ Stark D, Toncian T and Arefiev A 2016 Physical review letters 116185003 


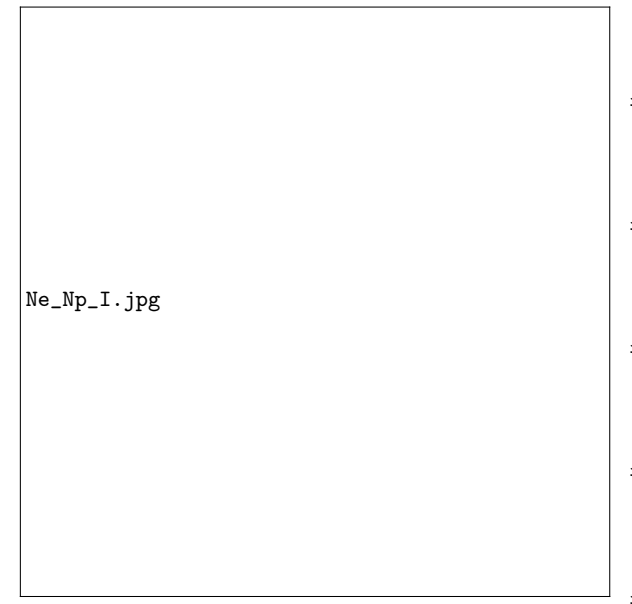

FIG. 10. The dependences of yield of photons $N_{\gamma}$ (a) (black solid line) and positrons $N_{e+}$ (b) on laser intensity. (a) draws the yield of photons with energy $E_{\gamma}>100 \mathrm{MeV}$ (blue dash line) as well. The red short dash line corresponds to the right $y$ axis, giving the averagese photons energy.

${ }^{3}$ Ridgers C, Brady C S, Duclous R, Kirk J, Bennett K, Arber T, Robinson $\mathrm{A}_{300}$ and Bell A 2012 Physical review letters 108165006

${ }^{4}$ Luo W, Wu S D, Liu W Y, Ma Y Y, Li F Y, Yuan T, Yu J Y, Chen M and

Sheng Z M 2018 Plasma Physics and Controlled Fusion 60095006

${ }^{5}$ Ridgers C, Brady C, Duclous R, Kirk J, Bennett K, Arber T and Bell A

2013 Physics of Plasmas 20056701

$345{ }^{6}$ Brady C, Ridgers C, Arber T and Bell A 2013 Plasma Physics and Controlled Fusion $\mathbf{5 5} 124016$

${ }^{7}$ Brady C S, Ridgers C, Arber T, Bell A and Kirk J 2012 Physical review letters 109245006

${ }^{8}$ Li H Z, Yu T P, Hu L X, Yin Y, Zou D B, Liu J X, Wang W Q, Hu S and Shao F Q 2017 Optics express 25 21583-21593

${ }^{9}$ Fuchs M, Weingartner R, Popp A, Major Z, Becker S, Osterhoff J, Cortrie I, Zeitler B, Hörlein R, Tsakiris G D et al. 2009 Nature physics 5826

${ }^{10}$ Kiselev S, Pukhov A and Kostyukov I 2004 Physical review letters 93 135004

$355{ }^{11}$ Phuoc K T, Corde S, Thaury C, Malka V, Tafzi A, Goddet J P, Shah R, Sebban S and Rousse A 2012 Nature Photonics 6308
${ }^{12}$ Yi L, Pukhov A, Luu-Thanh P and Shen B 2016 Physical review letters 116 115001

${ }^{13}$ Zou D, Pukhov A, Yi L, Zhuo H, Yu T, Yin Y and Shao F 2017 Scientific 360 reports 742666

${ }^{14}$ Ji L, Pukhov A, Kostyukov I Y, Shen B and Akli K 2014 Physical review letters 112145003 ${ }^{15}$ Zhu X L, Yu T P, Chen M, Weng S M and Sheng Z M 2018 New Journal of

${ }^{365}{ }^{16}$ Grismayer T, Vranic M, Martins J L, Fonseca R and Silva L 2016 Physics of Plasmas $\mathbf{2 3} 056706$

${ }^{17}$ Nerush E, Kostyukov I Y, Fedotov A, Narozhny N, Elkina N and Ruhl H 2011 Physical review letters 106035001

${ }^{18}$ Luo W, Liu W Y, Yuan T, Chen M, Yu J Y, Li F Y, Del Sorbo D, Ridgers C

70 and Sheng Z M 2018 Scientific reports 8 1-8

${ }^{19}$ Slade-Lowther C, Del Sorbo D and Ridgers C P 2019 New Journal of Physics 21013028

${ }^{20}$ Zamfir N 2014 The European Physical Journal Special Topics 2231221 1227

$375 \quad{ }^{21}$ Kostyukov I Y and Nerush E 2016 Physics of Plasmas 23093119

${ }^{22}$ Zhang Z, Teng J, Zhang B, Deng Z, He S, Cui B, Hong W, Zhou W and Gu Y 2018 Applied Physics Letters 113264101

${ }^{23}$ Nakamura T, Koga J K, Esirkepov T Z, Kando M, Korn G and Bulanov S V 2012 Physical review letters 108195001

${ }^{24}$ Wang W M, Sheng Z M, Gibbon P, Chen L M, Li Y T and Zhang J 2018 Proceedings of the National Academy of Sciences 115 9911-9916

${ }^{25}$ Erber T 1966 Reviews of Modern Physics 38626

${ }^{26}$ Katkov V, Strakhovenko V M 1998 Electromagnetic processes at high energies in oriented single crystals (World Scientific)

${ }^{27}$ Blackburn T G 2015 QED effects in laser-plasma interactions Ph.D. thesis Oxford University, UK

${ }^{28}$ Kirk J G, Bell A and Arka I 2009 Plasma Physics and Controlled Fusion 51085008

${ }^{29}$ Ritus V 1985 Journal of Russian Laser Research $6497-617$

${ }^{30}$ Uggerhøj U I 2005 Reviews of modern physics 771131

${ }^{31}$ Krajewska K and Kamiński J 2012 Physical Review A 85062102

${ }^{32}$ Krajewska K and Kamiński J 2012 Physical Review A 86052104

${ }^{33}$ Arber T, Bennett K, Brady C, Lawrence-Douglas A, Ramsay M, Sircombe N, Gillies P, Evans R, Schmitz H, Bell A et al. 2015 Plasma Physics and Controlled Fusion $\mathbf{5 7} 113001$

${ }^{34}$ Ridgers C P, Kirk J G, Duclous R, Blackburn T, Brady C, Bennett K, Arber $\mathrm{T}$ and Bell A 2014 Journal of computational physics $\mathbf{2 6 0} 273-285$

${ }^{35}$ Bulanov S, Esirkepov T Z, Kando M, Koga J, Kondo K and Korn G 2015 Plasma Physics Reports $\mathbf{4 1} 1-51$

${ }^{36}$ Stenson E, Horn-Stanja J, Stoneking M and Pedersen T S 2017 Journal of Plasma Physics $\mathbf{8 3}$

${ }^{37}$ Di Piazza A, Hatsagortsyan K and Keitel C H 2010 Physical review letters 105220403

${ }^{38}$ Ruffini R, Vereshchagin G and Xue S S 2010 Physics Reports 487 1-140 ${ }^{39}$ Piran T 2005 Reviews of Modern Physics 761143 


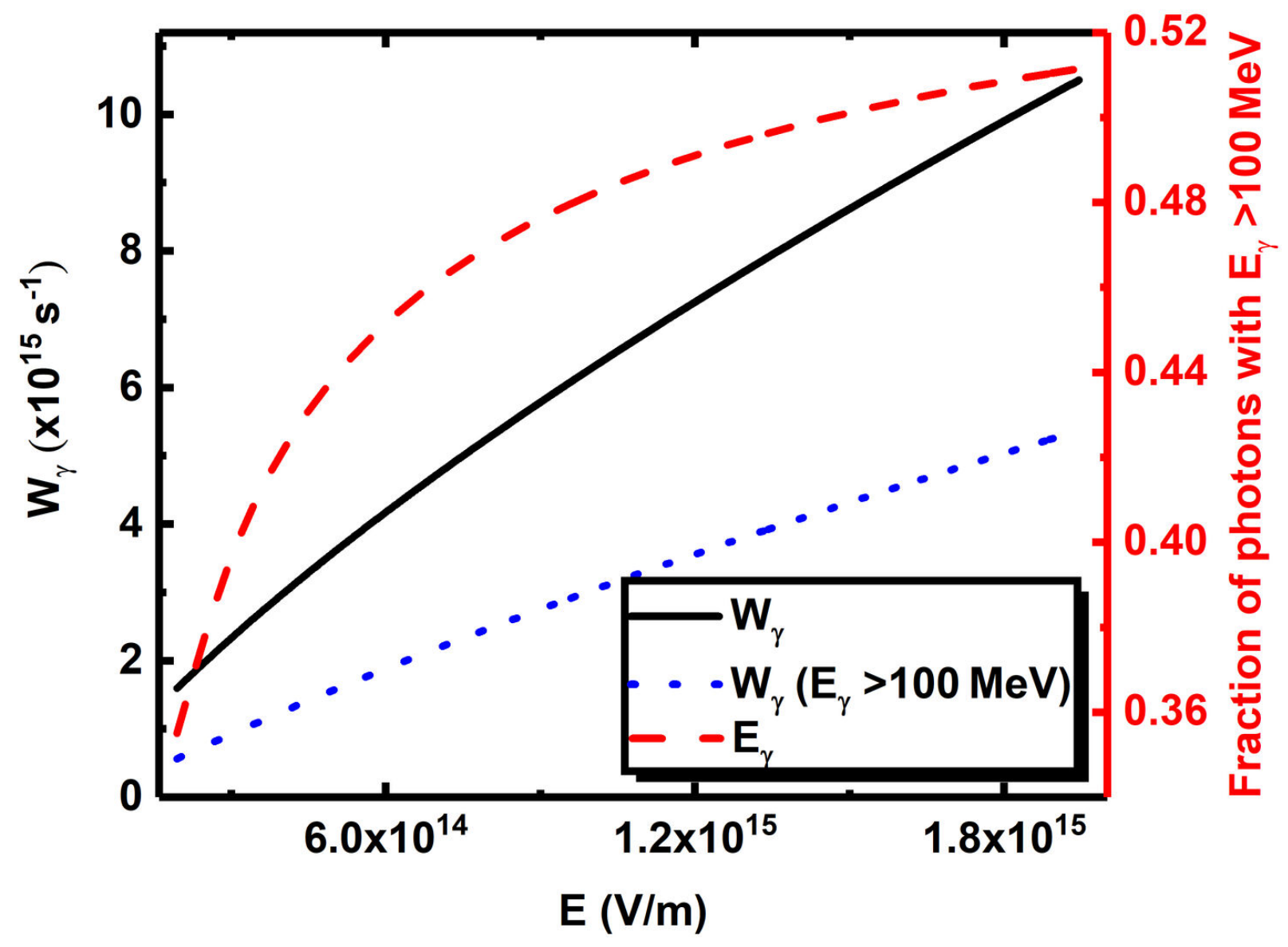




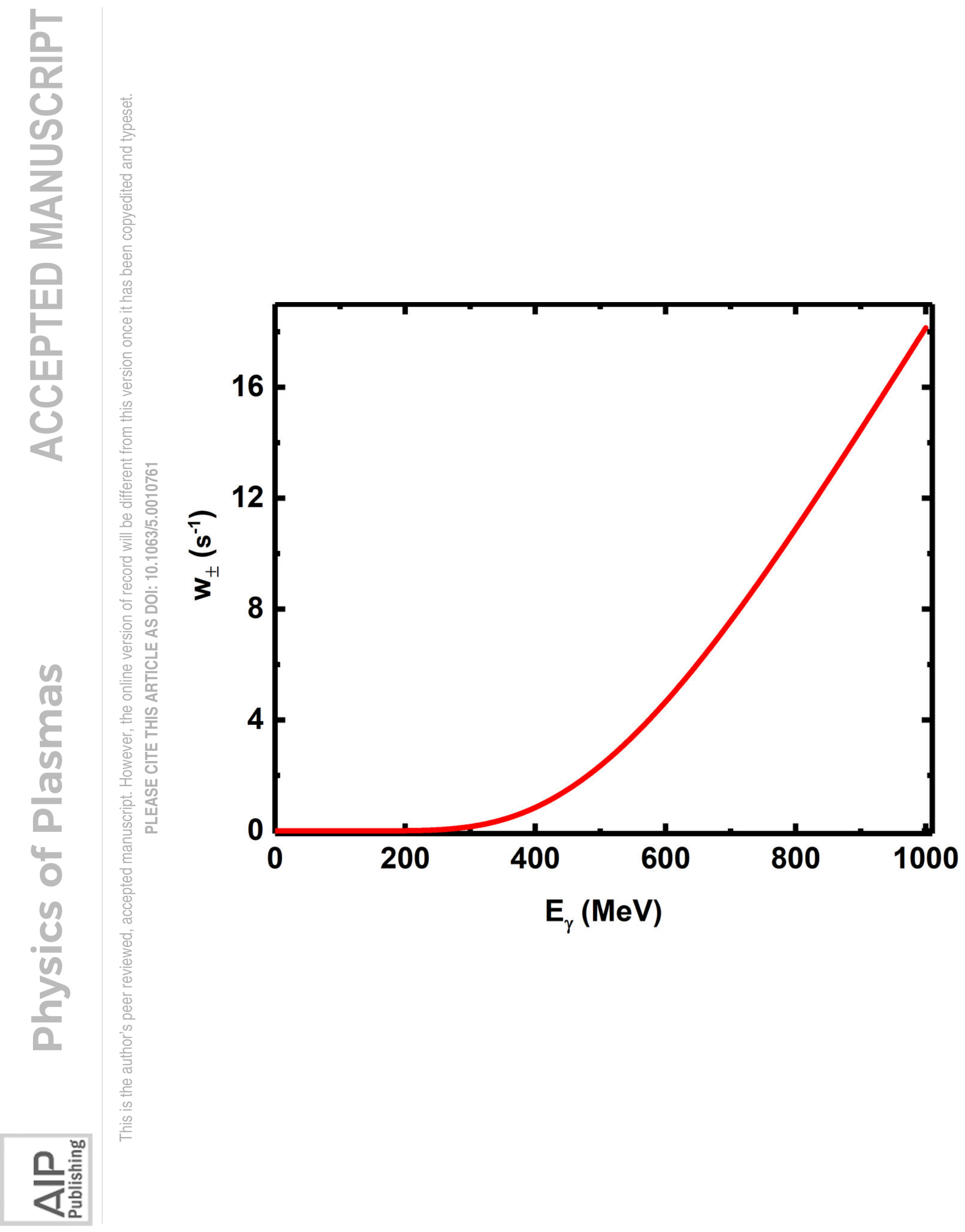




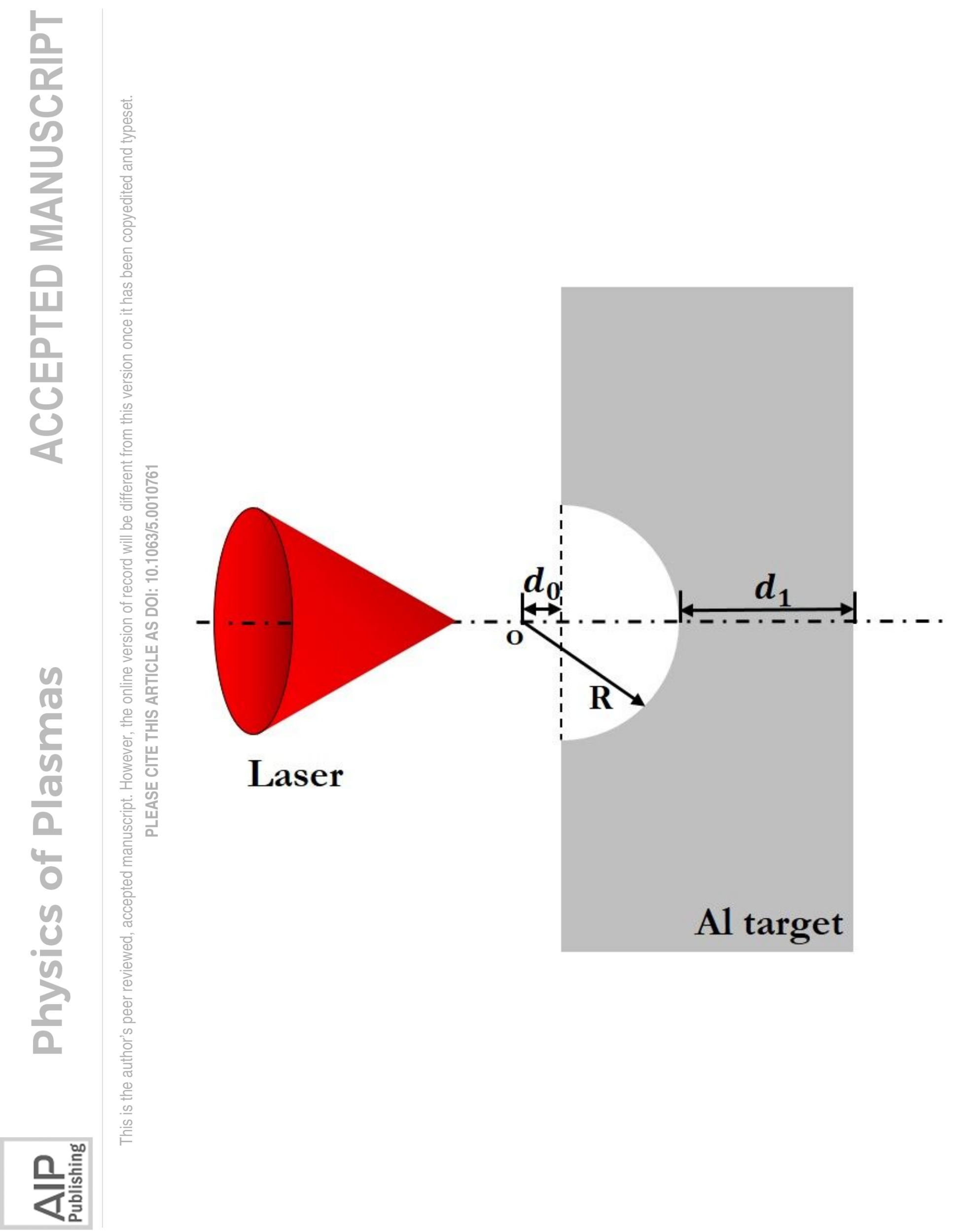




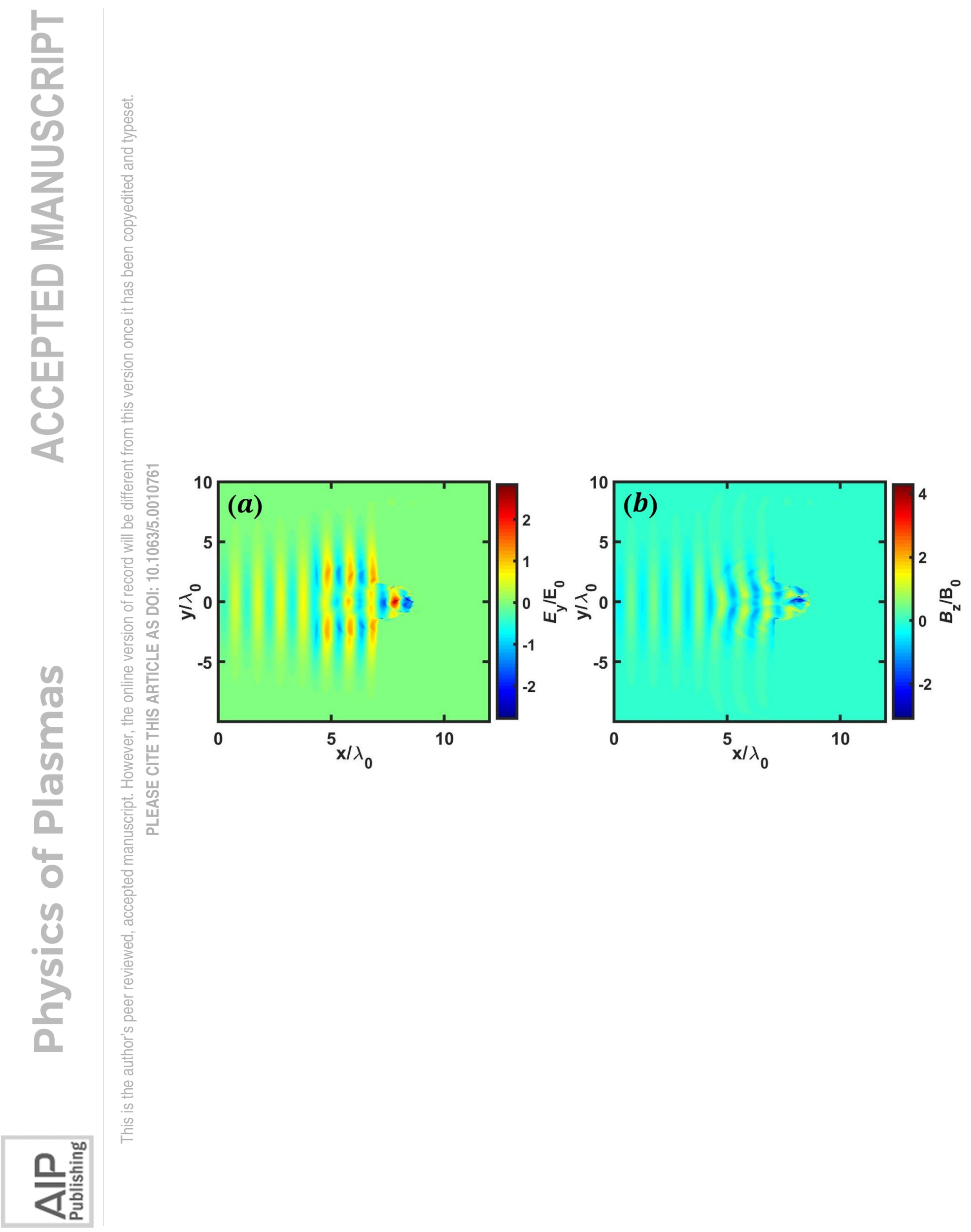



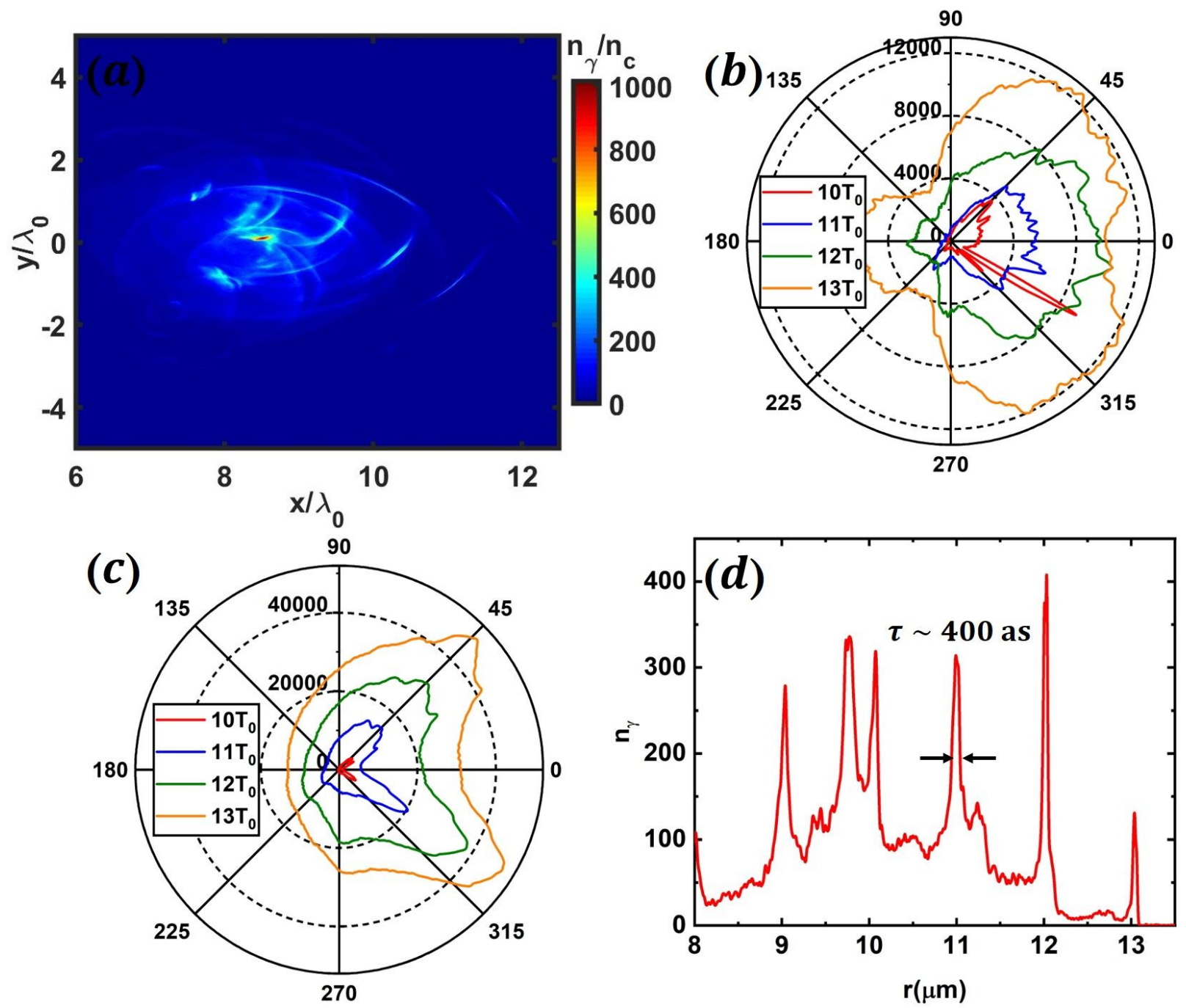


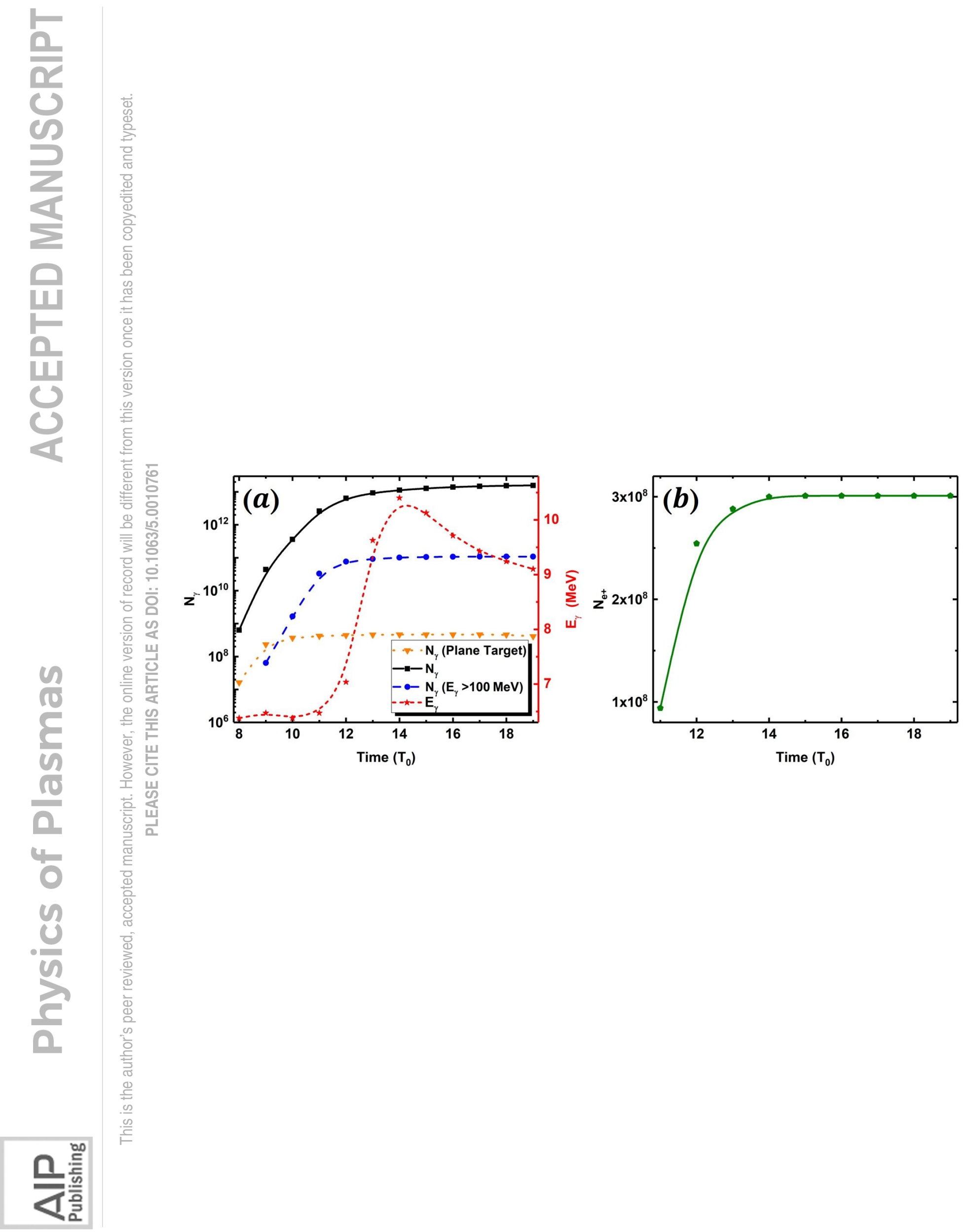



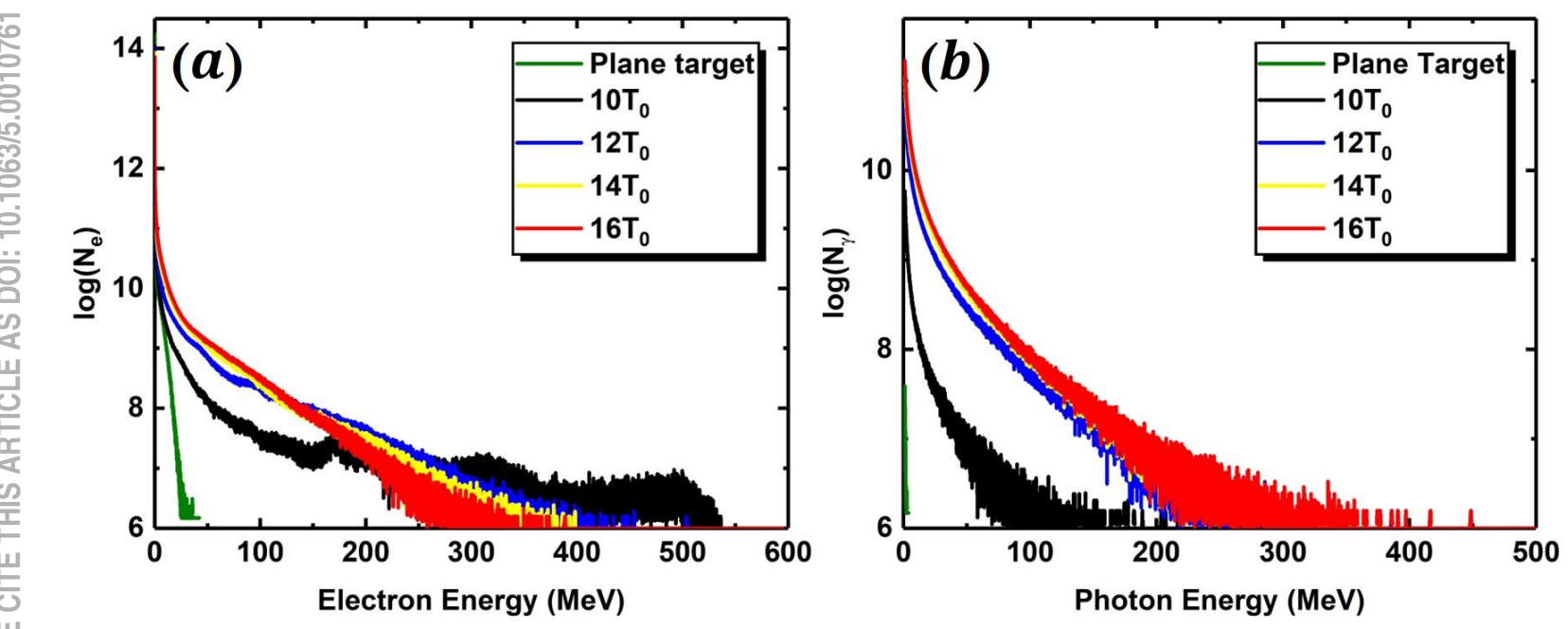


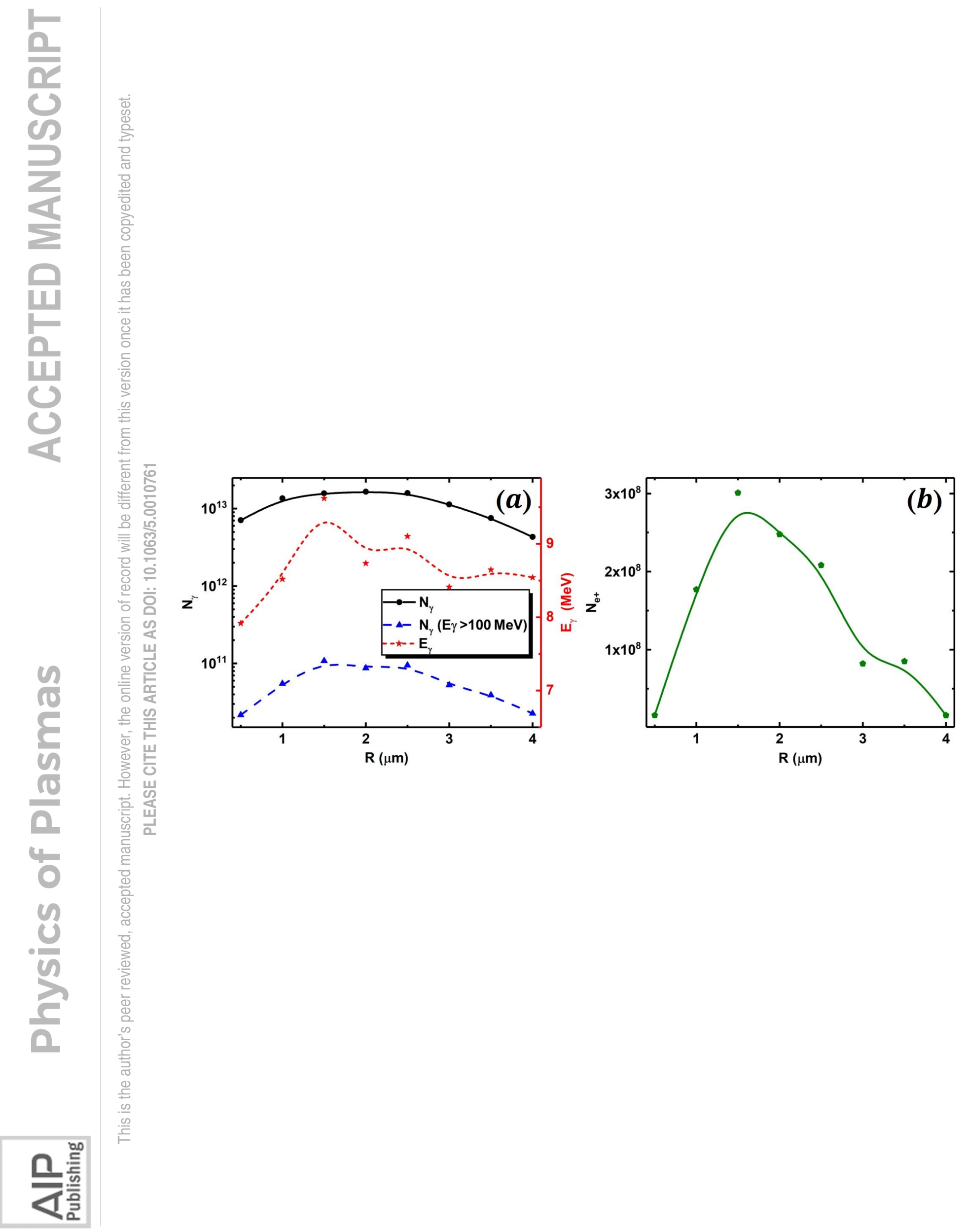




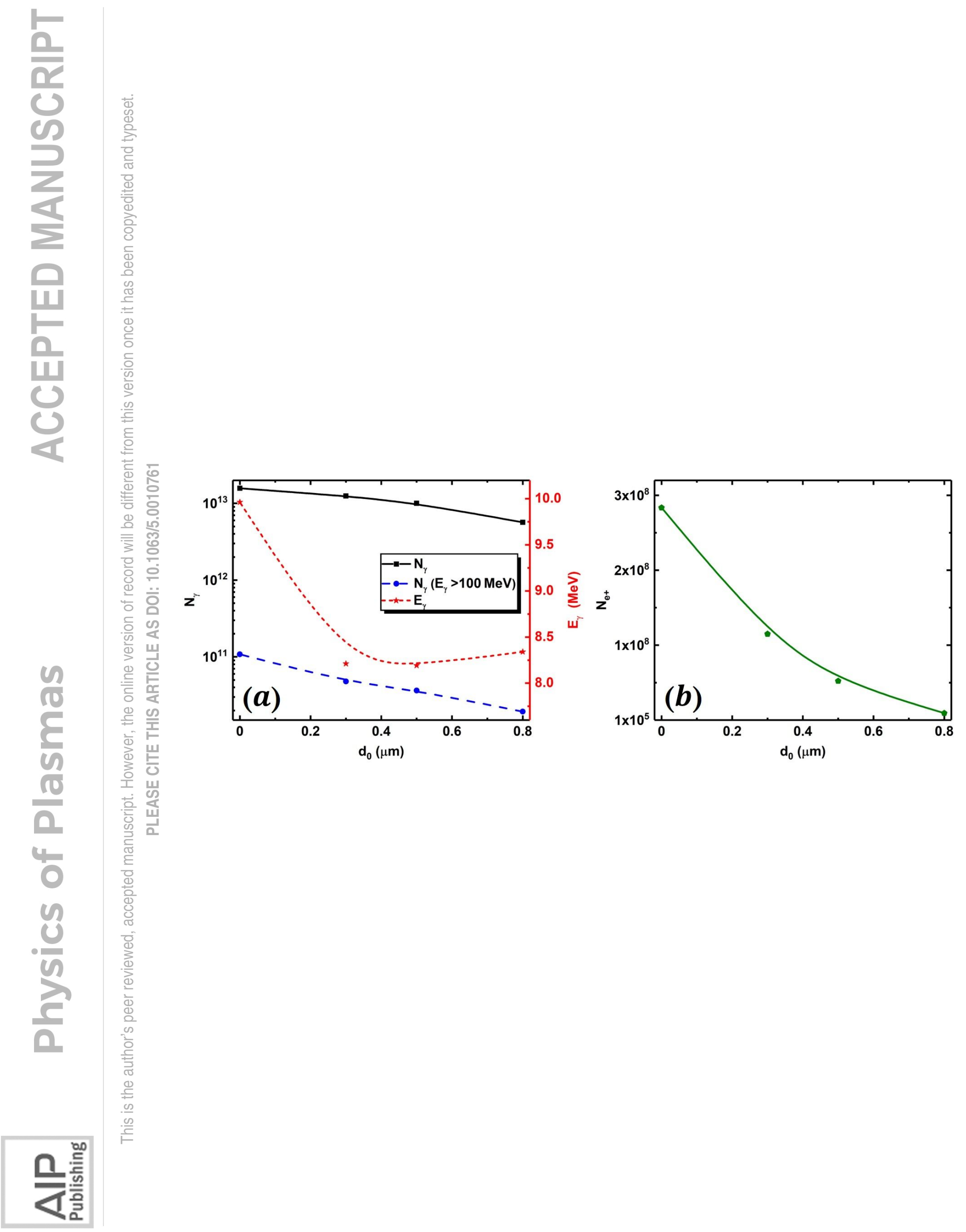




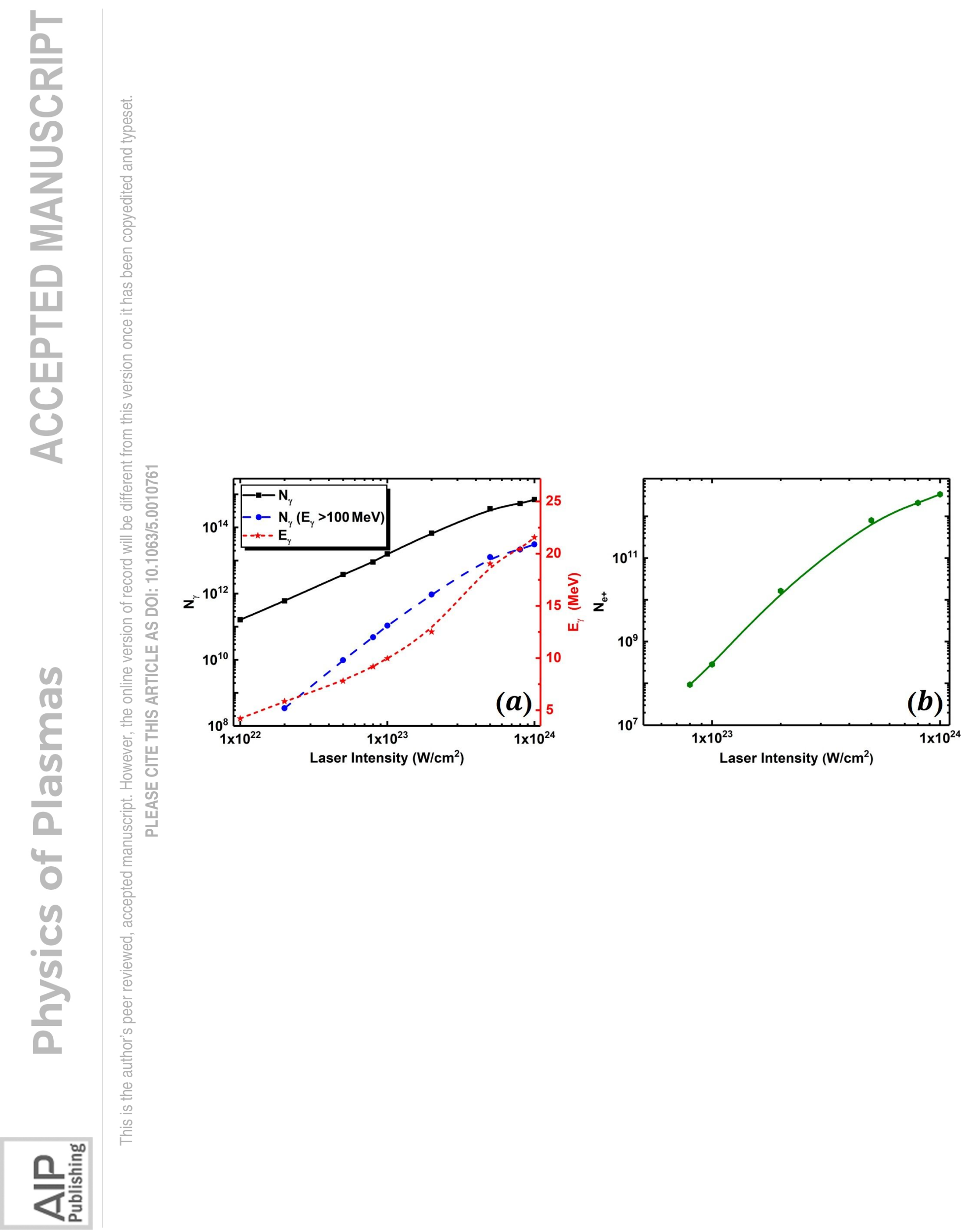

